\title{
FUNCTIONAL EQUATIONS OF ITERATED INTEGRALS WITH REGULAR SINGULARITIES
}

\section{ZDZISŁAW WOJTKOWIAK}

\section{§0. Introduction}

Polylogarithmic functions satisfy functional equations. The most famous equation is of course the functional equation of the logarithm

$$
\log x+\log y=\log (x \cdot y) .
$$

The other well known equation is the Abel equation of the dilogarithm

$$
\begin{aligned}
\operatorname{Li}_{2}\left(\frac{x}{1-x} \cdot \frac{y}{1-y}\right)= & \operatorname{Li}_{2}\left(\frac{y}{1-x}\right)+\operatorname{Li}_{2}\left(\frac{x}{1-y}\right)-\mathrm{Li}_{2}(x)-\mathrm{Li}_{2}(y) \\
& -\log (1-x) \log (1-y)
\end{aligned}
$$

Polylogarithms are special cases of more general iterated integrals. One can hope that the known results about functional equations of polylogarithms hold also for more general iterated integrals. In fact in [5] we have proved some general results about functional equations of iterated integrals on $P^{1}(\mathbf{C})$ minus several points. In this paper we generalize our results from [5] to functional equations of iterated integrals on any smooth, quasi-projective algebraic variety.

Our principal tool is the universal unipotent connection with logarithmic singularities. First we prove our results for a complement of a divisor with normal crossings in a smooth, projective variety. Next, using results of Hironaka about resolution of singularities we extend our results to smooth, quasi-projective varieties. The proofs (for a complement of a divisor with normal crossings) are straightforward generalizations of methods from [5]. These results are in the first three sections of this paper.

In the fourth section of the paper we are dealing with the dilogarithm. It is well known that any functional equation of the logarithm on $P^{1}(\mathbf{C})$ can be obtained by successive applications of the functional equation

Received March 2, 1994. 


$$
\log x+\log y=\log (x \cdot y)
$$

We shall show that there is a similar situation for the dilogarithm. This section is a natural complement of our previous work [5]. The result was presented in the Strasbourg Conference on Algebraic $K$-theory 1992. In the last section we give a sufficient and necessary condition to have a functional equation of iterated integrals on a pointed projective line in terms of exotic analogues of the Bloch group for the dilogarithm.

\section{§1. Canonical connection with logarithmic singularities}

Let $X$ be a smooth, projective scheme of finite type over a field $k$ of characteristic zero. Let $D$ be a divisor with normal crossings in $X$ and let $V=X \backslash D$. Let

$$
A^{*}(V):=\Gamma\left(X, \Omega_{X}^{*}\langle\log D\rangle\right)
$$

be a differential algebra of global sections of the algebraic De Rham complex on $X$ with logarithmic singularities along $D$.

1.1. It follows from [1] Corollaire 3.2.14 that each element of $A^{*}(V)$ is closed and the natural map $A^{*}(V) \rightarrow H_{D R}^{*}(V)$ is injective.

We shall denote by $\wedge^{2}\left(A^{1}(V)\right)$ the exterior product of the vector space $A^{1}(V)$ with itself and by $A^{1}(V) \wedge A^{1}(V)$ the image of $\wedge^{2}\left(A^{1}(V)\right)$ in $A^{2}(V)$.

Let $H(V):=\left(A^{1}(V)\right)^{*}$ and $R(V):=\left(A^{1}(V) \wedge A^{1}(V)\right)^{*}$ be dual vector spaces. The surjective map $\wedge^{2}\left(A^{1}(V)\right) \rightarrow A^{1}(V) \wedge A^{1}(V)$ induces the injective $\operatorname{map} R(V) \rightarrow \wedge^{2}(H(V))$.

Let $A$ be a commutative ring with 1 , and let $M$ be an $A$-module. We say that an $A$-module Lie $(M)$ is a free Lie algebra (over $A$ ) on the $A$-module $M$ if:

i) there is an $A$-module homomorphism $\mathscr{C}_{M}: M \rightarrow \operatorname{Lie}(M)$,

ii) for any Lie algebra $L$ over $A$ and any $A$-linear homomorphism $f: M \rightarrow L$ there is a unique $A$-linear Lie algebra homomorphism $\bar{f}: \operatorname{Lie}(M) \rightarrow L$ such that $f=\bar{f} \circ \mathscr{C}_{M}$.

The uniqueness and the existence of $\operatorname{Lie}(M)$ is a standard exercise in linear algebra. We point out that a free Lie algebra on $n$ free generators is a free Lie algebra on the abelian group $\mathbf{Z}^{n}$.

Let Lie $(H(V))$ be a free Lie algebra over $k$ on $H(V)$. Observe that $R(V)$ is contained in degree 2 terms of Lie $(H(V))$. Let $(R(V))$ be a Lie ideal generated by $R(V)$. We set 


$$
\operatorname{Lie}(V):=\operatorname{Lie}(H(V)) /(R(V))
$$

and

$$
\mathrm{L}(V):=\underset{\leftarrow}{\lim }\left(\operatorname{Lie}(V) / \Gamma^{n}(V)\right),
$$

where $\Gamma^{2} \operatorname{Lie}(V):=[\operatorname{Lie}(V), \operatorname{Lie}(V)]$ and $\Gamma^{n+1} \operatorname{Lie}(V):=\left[\Gamma^{n} \operatorname{Lie}(V) ; \operatorname{Lie}(V)\right]$.

The Lie algebra $L(V)$ we equipped with the multiplication given by the Baker-Campbell-Hausdorff formula and the obtained group we shall denote by $\pi(V)$. Its Lie algebra can be identified with $L(V)$. We define a one form $\omega_{V}$ on $V$ with values in the Lie algebra $L(V)$ in the following way. The form $\omega_{V}$ corresponds to the identity homomorphism $\operatorname{id}_{A^{1}(V)}$ under the natural isomorphism

$$
\left.A^{1}(V) \otimes H(V)=A^{1}(V) \otimes\left(A^{1}(V)\right)^{*} \approx \operatorname{Hom}\left(A^{1}(V), A^{1}(V)\right)\right) .
$$

Lemma 1.2. The one-form $\omega_{V}$ is integrable.

Proof. It is sufficient to show that $d \omega_{V}+\frac{1}{2}\left[\omega_{V}, \omega_{V}\right]=0$. It follows from 1.1 that $d \omega_{V}=0$. Let $K:=\operatorname{ker}\left(\wedge^{2} A^{1}(V) \rightarrow A^{1}(V) \wedge A^{1}(V)\right)$. The two-form $\left[\omega_{V}, \omega_{V}\right]$ is represented by the map $K \rightarrow \wedge^{2}\left(A^{1}(V)\right) \rightarrow A^{1}(V) \wedge A^{1}(V)$, hence it is zero.

Let us assume that $k$ is the field of complex numbers $\mathbf{C}$. Then $V$ is a complex variety with the standard complex topology.

Let $x, z \in V$ be two points in $V$ and let $\gamma$ be a smooth path in $V$ from $x$ to $z$.

The principal $\pi(V)$-bundle $V \times \pi(V) \rightarrow V$ we equip with the connection (integrable by Lemma 1.2$)$ given by the form $\omega_{V}$. Let $\left(\gamma(z), L_{V}(z ; x, \gamma)\right)$ be its horizontal section along $\gamma$ such that the value of $L_{V}(z ; x, \gamma)$ at the starting point is 0 .

Definition 1.3. Let $x \in V$ and let $\alpha \in \pi_{1}(V, x)$ be a loop. We shall define a homomorphism

$$
\theta_{x, V}: \pi_{1}(V, x) \rightarrow \pi(V)
$$

by the formula

$$
\theta_{x, V}(\alpha):=L_{V}(\alpha(1) ; x, \alpha)
$$

and we call it the monodromy homomorphism of the form $\omega_{V}$ (at the point $x$ ).

1.4. Let $X_{i}$ (for $i=1,2$ ) be smooth, projective schemes of finite type over $k$. Let $D_{i}$ be divisors with normal crossings in $X_{i}$ and let $V_{i}=X_{i} \backslash D_{i}$ for $i=1,2$. 
Let $f: X_{1} \rightarrow X_{2}$ be a morphism such that $f^{-1}\left(D_{2}\right) \subset D_{1}$. Then $f$ induces a morphism from $V_{1}$ to $V_{2}$ which we denote also by $f$. Moreover $f$ induces $f^{*}: A^{1}\left(V_{2}\right) \rightarrow$ $A^{1}\left(V_{1}\right)$.

Let $f_{*}: H\left(V_{1}\right) \rightarrow H\left(V_{2}\right)$ be the dual map. The map $f_{*}$ induces a group homomorphism

$$
f_{*}: \pi\left(V_{1}\right) \rightarrow \pi\left(V_{2}\right)
$$

Lemma 1.5. We have

$$
f_{*}\left(\omega_{V_{1}}\right)=f^{*}\left(\omega_{V_{2}}\right)
$$

Corollary 1.6. Assume that $k$ is the field of complex numbers. Then we have

$$
f^{*}\left(L_{V_{1}}(z ; x, \gamma)\right)=L_{V_{2}}(f(z) ; f(x), f(\gamma)) \text {. }
$$

The lemma follows from the definition of $\omega_{V}$ as $\operatorname{id}_{A^{1}(V)}$. The corollary is the direct consequence of the lemma.

\section{Functional equations I}

2.0. Let $X$ and $Y$ be smooth, projective schemes of finite type over C. Let $D$ and $E$ be divisors with normal crossings in $X$ and $Y$ respectively. Let us set $U=$ $X \backslash D$ and $V=Y \backslash E$. Let $\operatorname{Alg}(\pi(U))$ and $\operatorname{Alg}(\pi(V))$ be algebras of regular func tions on $\pi(U)$ and $\pi(V)$ respectively. The map $f: X \rightarrow Y$ such that $f^{-1}(E) \subset D$ determines a map from $U$ to $V$, which we shall denote also by $f$.

THEOREM 2.1. Let $f_{1}, \ldots, f_{N}: X \rightarrow Y$ be morphisms such that $f_{i}^{-1}(E) \subset D$ for each $i$. Let $\varphi_{1}, \ldots, \varphi_{N} \in \operatorname{Alg}(\pi(V))$ and let $p\left(t, \ldots, t_{N}\right)$ be a polynomial in variables $t_{1}, \ldots, t_{N}$. Then there is a functional equation

1) $\quad p\left(\varphi_{1}\left(L_{V}\left(f_{1}(z) ; f_{1}(x), f_{1}(\gamma)\right)\right), \ldots, \varphi_{N}\left(L_{V}\left(f_{N}(z) ; f_{N}(x), f_{N}(\gamma)\right)\right)=0\right.$

if and only if

2)

$$
p\left(\varphi_{1} \circ\left(f_{1}\right)_{*}, \ldots, \varphi_{N} \circ\left(f_{N}\right)_{*}\right)=0
$$

in $\operatorname{Alg}(\pi(U))$.

Proof. The equation 1) follows from 2) by Corollary 1.6. The monodromy representation $\pi_{1}(U, x) \rightarrow \pi(U)^{a b}$ induced by $\theta_{x, U}$ has a Zariski dense image in $\pi(U)^{a b}$, hence the monodromy representation has a Zariski dense image in $\pi(U)$ / 
$\Gamma^{n} \pi(U)$ for any $n$. Hence the function $p\left(\varphi_{1} \circ\left(f_{1}\right)_{*}, \ldots, \varphi_{N} \circ\left(f_{N}\right)_{*}\right)$ vanishes on a Zariski dense subset of $\pi(U) / \Gamma^{n} \pi(U)$. If $n$ is big enough this implies that the function $p\left(\varphi_{1} \circ\left(f_{1}\right)_{*}, \ldots, \varphi_{N} \circ\left(f_{N}\right)_{*}\right)$ is the zero function.

Let $\operatorname{Lie}(V)^{*}:=\underset{n}{\lim }\left(\operatorname{Lie}(V) / \Gamma^{n} \operatorname{Lie}(V)\right)^{*}$ be the direct limit of dual vector spaces. Observe that $\operatorname{Lie}(V)^{*}$ has a natural grading, $\operatorname{Lie}(V)^{*}=$ $\bigoplus_{n=1}^{\infty}\left(\operatorname{Lie}(V)^{*}\right)_{n}$ where $\left(\operatorname{Lie}(V)^{*}\right)_{n}=\left(\Gamma^{n} \operatorname{Lie}(V) / \Gamma^{n+1} \operatorname{Lie}(V)\right)^{*}$. The elements of $\operatorname{Lie}(V)^{*}$ can be view as elements of $\operatorname{Alg}(\pi(V))$. In fact $\operatorname{Lie}(V)^{*}$ generates $\operatorname{Alg}(\pi(V))$ as a $\mathbf{C}$-algebra.

Corollary 2.2. Let $f_{1}, \ldots, f_{N}$ be as in Theorem 2.1. Let $n_{1}, \ldots, n_{N}$ be complex numbers and let $v_{1}^{*}, \ldots, v_{N}^{*} \in\left(\operatorname{Lie}(V)^{*}\right)_{n}$. Then there is a functional equation

$$
\sum_{i=1}^{N} n_{i} \cdot v_{i}^{*}\left(L_{V}\left(f_{i}(z) ; f_{i}(x), f_{i}(\gamma)\right)=0\right.
$$

if and only if

$$
\sum_{i=1}^{N} n_{i} \cdot v_{i}^{*} \circ\left(f_{i}\right)_{*}=0
$$

in the group $\operatorname{Hom}\left(\Gamma^{n} \pi(U) / \Gamma^{n+1} \pi(U) ; \mathbf{C}\right)$, where $\left(f_{i}\right)_{*}: \Gamma^{n} \pi(U) / \Gamma^{n+1} \pi(U) \rightarrow$ $\Gamma^{n} \pi(V) / \Gamma^{n+1} \pi(V)$ are homomorphisms induced by $\left(f_{i}\right)_{*}: \pi(U) \rightarrow \pi(V)$.

The corollary is a special case of Theorem 2.1.

The monodromy homomorphism $\theta_{y, V}: \pi_{1}(V, y) \rightarrow \pi(V)$ induces a homomorphism $\theta_{V}^{(n)}: \Gamma^{n} \pi_{1}(V, y) / \Gamma^{n+1} \pi_{1}(V, y) \rightarrow \Gamma^{n} \pi(V) / \Gamma^{n+1} \pi(V)$, which has a Zariski dense image and does not depend on a base point $y$. For any $v_{i}^{*} \in$ $\left(\operatorname{Lie}(V)^{*}\right)_{n}$ the composition $v_{i}^{*} \circ \theta_{V}^{(n)}$ we shall denote by $V_{i}^{*}$. Let $\left(f_{i}\right)_{\#}: \Gamma^{n} \pi_{1}(U, x)$ $/ \Gamma^{n+1} \pi_{1}(U, x) \rightarrow \Gamma^{n} \pi_{1}(V, y) / \Gamma^{n+1} \pi_{1}(V, y)$ be the map induced by $f_{i}: U \rightarrow V$.

CoRollary 2.3. Let $f_{1}, \ldots, f_{N}, n_{1}, \ldots, n_{N}$ and $v_{1}^{*}, \ldots, v_{N}^{*}$ be as in Corollary 2.2. Then there is a functional equation

$$
\sum_{i=1}^{N} n_{i} \cdot v_{i}^{*}\left(L_{V}\left(f_{i}(z) ; f_{i}(x), f_{i}(\gamma)\right)=0\right.
$$

if and only if

$$
\sum_{i=1}^{N} n_{i} \cdot V_{i}^{*} \circ\left(f_{i}\right)_{\#}=0
$$


in the group $\operatorname{Hom}\left(\Gamma^{n} \pi_{1}(U, x) / \Gamma^{n+1} \pi_{1}(U, x) ; \mathbf{C}\right)$.

The Corollary 2.3 follows from Corollary 2.2 , the equality $\left(f_{i}\right)_{*} \circ \theta_{U}^{(n)}=\theta_{V}^{(n)}$ 。 $\left(f_{i}\right)_{\#}$ (which is a consequence of Corollary 1.6) and from the fact that $\operatorname{im}\left(\theta_{U}^{(n)}\right)$ is Zariski dense in $\Gamma^{n} \pi(U) / \Gamma^{n+1} \pi(U)$.

Assume that $X$ and $Y$ are defined over a field $k$ contained in the field of complex numbers. Let $D$ and $E$ be divisors with normal crossings in $X$ and $Y$ respectively. Let $U=X \backslash D$ and $V=Y \backslash E$.

Setting ()$_{\mathbf{C}}:=() \times \operatorname{Spec} \mathbf{C}$ we find ourselves in the familiar situation over $\mathbf{C}$.

Notice that $\operatorname{Lie}(U)$ and $\operatorname{Lie}(V)$ are Lie algebras over $k,\left(\operatorname{Lie}()^{*}\right)_{n}=$ $\operatorname{Hom}\left(\Gamma^{n} \operatorname{Lie}() / \Gamma^{n+1} \operatorname{Lie}() ; k\right)$ for ()$=(U)$ or $(V)$. We have $\left(\operatorname{Lie}(V)^{*}\right)_{n} \otimes_{k}$ $\mathbf{C}=\left(\operatorname{Lie}\left(V_{\mathbf{C}}\right)^{*}\right)_{n}$. We shall identify $v^{*} \in\left(\operatorname{Lie}(V)^{*}\right)_{n}$ with its image $v^{*} \otimes 1$ in $\left(\operatorname{Lie}\left(V_{\mathbf{C}}\right)^{*}\right)$

COROLlary 2.4. Let $f_{1}, \ldots, f_{N}: X \rightarrow Y$ be morphisms such that $f_{i}^{-1}(E) \subset D$ for each $i$. Let $v_{1}^{*}, \ldots, v_{N}^{*} \in\left(\operatorname{Lie}(V)^{*}\right)$ and let $c_{1}, \ldots, c_{N}$ be complex numbers not all equal to zero. If there is a functional equation of the form

$$
\sum_{i=1}^{N} c_{i} \cdot v_{i}^{*}\left(L_{V_{\mathbf{C}}}\left(f_{i}(z) ; f_{i}(x), f_{i}(\gamma)\right)\right)=0
$$

then there are $q_{1}, \ldots, q_{N} \in k$ not all equal to zero such that

$$
\sum_{i=1}^{N} q_{i} \cdot v_{i}^{*}\left(L_{V_{\mathrm{C}}}\left(f_{i}(z) ; f_{i}(x), f_{i}(\gamma)\right)\right)=0 .
$$

Corollary 2.4 follows from Corollary 2.2 and from the well known fact that if vectors $w_{1}, \ldots, w_{N} \in k^{r}$ are linearly dependent in $k^{r} \underset{k}{\otimes} \mathbf{C} \approx \mathbf{C}^{r}$ then they are already linearly dependent in $k^{r}$.

Observe that in [5] Corollary 10.6 .7 we have that $q_{1}, \ldots, q_{N}\left(c_{1}, \ldots, c_{N}\right.$ in the notation of [5]) are in $\mathbf{Q}$ even if the varieties are defined over any finitely generated extension of $\mathbf{Q}$ in $\mathbf{C}$. Below there is a kind of formal analogue.

We consider the case when $X, D$ and $U$, and $Y, E$ and $V$ are over $k$. There is a natural map

$$
\int: A^{1}(V) \rightarrow \operatorname{Hom}\left(H_{1}\left(V_{\mathbf{C}}, \mathbf{Q}\right) ; \mathbf{C}\right)
$$

given by $\left(\int \omega\right)(\gamma):=\int_{r} \omega$. 
Let $\mathbf{Q}^{1}(V):=\left\{\omega \in A^{1}(V) \mid\left(\int \omega\right)(\gamma) \in \mathbf{Q} \cdot(-2 \pi i), \forall \gamma \in H_{1}\left(V_{\mathbf{C}}, \mathbf{Q}\right\}\right\}$. Then $\mathbf{Q}^{1}(V)$ is a $\mathbf{Q}$-lattice in $A^{1}(V)$ i.e. the natural map $\mathbf{Q}^{1}(V) \otimes k \rightarrow A^{1}(V)$ is injective. Observe that for any $f: X \rightarrow Y$ such that $f^{-1}(E) \subset D$ we have $f^{*}\left(\mathbf{Q}^{1}(V)\right) \subset \mathbf{Q}^{1}(U)$.

Let $0 \rightarrow\left(\mathbf{Q}^{1}(V) \wedge \mathbf{Q}^{1}(V)\right)^{*} \gg \wedge^{2}\left(\mathbf{Q}^{1}(V)^{*}\right)$ be the dual map of

$$
\stackrel{2}{\wedge}\left(\mathbf{Q}^{1}(V)\right) \rightarrow \mathbf{Q}^{1}(V) \wedge \mathbf{Q}^{1}(V) \rightarrow 0 \text {. }
$$

Let $\operatorname{Lie}_{2 \pi i}(V)$ be a quotient of a free Lie algebra over $\mathbf{Q}$ on $\mathbf{Q}^{1}(V)_{*}$ by the Lie ideal generated by $\left(\mathbf{Q}^{1}(V) \wedge \mathbf{Q}^{1}(V)\right)^{*}$. The inclusion $\mathbf{Q}^{1}(V) \otimes k \rightarrow A^{1}(V)$ induces a surjective morphism of Lie algebras $\operatorname{Lie}(V) \rightarrow \operatorname{Lie}_{2 \pi i}(V) \otimes k$. Hence we have an inclusion

$$
\operatorname{Hom}\left(\Gamma^{n} \operatorname{Lie}_{2 \pi i}(V) / \Gamma^{n+1} \operatorname{Lie}_{2 \pi i}(V) ; \mathbf{Q}\right) \otimes k \rightarrow\left(\operatorname{Lie}(V)^{*}\right)_{n} .
$$

COROLlary 2.5. Let $f_{1}, \ldots, f_{N}: X \rightarrow Y$ be morphisms such that $f_{i}^{-1}(E) \subset D$ for each $i$. Let $v_{1}^{*}, \ldots, v_{N}^{*} \in \operatorname{Hom}\left(\Gamma^{n} \operatorname{Lie}_{2 \pi i}(V) / \Gamma^{n+1} \operatorname{Lie}_{2 \pi i}(V) ; \mathbf{Q}\right)$ and let $c_{1}, \ldots, c_{N}$ be complex numbers not all equal to zero. If there is a functional equation of the form

$$
\sum_{i=1}^{N} c_{i} \cdot v_{i}^{*}\left(L_{V_{\mathbf{C}}}\left(f_{i}(z) ; f_{i}(x), f_{i}(\gamma)\right)=0\right.
$$

then there are rational numbers $q_{1}, \ldots, q_{N}$ not all equal to zero such that

$$
\sum_{i=1}^{N} q_{i} \cdot v_{i}^{*}\left(L_{V_{\mathbf{C}}}\left(f_{i}(z) ; f_{i}(x), f_{i}(\gamma)\right)\right)=0
$$

The proof of the corollary is the same as the proof of Corollary 2.4.

\section{Functional equations II}

Now we shall study functional equations on any $U$ smooth, quasi-projective over Spec C. Following Hironaka [3] we can find $X$ smooth, projective, such that $U \subset X$ and $X \backslash U$ is a divisor with normal crossings. Hence we are in the situation of 2.0 .

Let us assume that $U$ and $V$ are smooth, quasi-projective. Let $f_{1}, \ldots, f_{N}: U \rightarrow$ $V$ be morphisms of schemes over $\mathbf{C}$. Let $X^{\prime}$ and $Y$ be smooth, projective compactifications of $U$ and $V$ such that $X^{\prime} \backslash U$ and $Y \backslash V$ are divisors with normal crossings. Let $F: U \rightarrow X^{\prime} \times Y \times \cdots \times Y$ be given by $F(u)=\left(u, f_{1}(n), \ldots, f_{N}(u)\right)$. Let $X$ be a resolution of singularities of the closure of the image of $U$ in $X^{\prime} \times Y$ 
$\times \cdots \times Y$. Then for any $i$ we have a morphism $\bar{f}_{i}: X \rightarrow Y$, induced by the projection on the $i$-th $Y$ factor, making the diagram

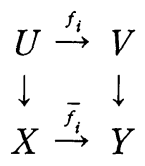

commutative.

Now the assumptions from Section 2 (in Theorem 2.1, in Corollary 2.2, ..) about morphisms $f_{1}, \ldots, f_{n}$ are satisfied. Hence we get the following theorem.

Theorem 3.1. All results from Section 2 (Theorem 2.1, Corollaries 2.2-2.5) hold for $U$ and $V$ smooth and quasi-projective over a field $k$ of characteristic zero and $f_{1}, \ldots$, $f_{N}: U \rightarrow V$ morphisms of schemes over Spec $k$.

To show Corollary 2.4 in general situation (and also Corollary 2.5 for any $k$ in $\mathbf{C}$ ), one must notice that $X$ and $Y, U \hookrightarrow X, X, V \hookrightarrow Y$ and $\bar{f}_{i}$ are over Spec $k$ if $U, V$ and $f_{i}$ are over Spec $k$. In all other cases it is sufficient to work over $\mathbf{C}$.

Remark. Theorem F from [5] is a special case of Theorem 3.1.

\section{Functional equations of the dilogarithm}

In this section we want to discuss some elementary properties of the dilogarithm. It is well known that any functional equation of the logarithm on $P^{1}(\mathbf{C})$ can be obtained by successive applications of the functional equation

$$
\log x+\log y=\log (x \cdot y) \text {. }
$$

We shall show that there is a similar situation for the dilogarithm. Of course we must replace the equation (1) by a variant of Abel-Spencer equation. We shall use a form due to Hill (see [4] 1.18 or [2])

$$
\begin{aligned}
\mathrm{Li}_{2}(x \cdot y)=\mathrm{Li}_{2}(x)+ & \mathrm{Li}_{2}(y)+\mathrm{Li}_{2}\left(x \cdot \frac{1-y}{x-1}\right)+\mathrm{Li}_{2}\left(y \cdot \frac{1-x}{y-1}\right) \\
& +\frac{1}{2} \log ^{2}\left(\frac{1-x}{1-y}\right) .
\end{aligned}
$$

Let $A_{2}(\mathbf{C}(z)):=\mathbf{Z}(\mathbf{C}(z) \backslash\{0,1\})$ be a free group on the set $\mathbf{C}(z) \backslash\{0,1\}$. Elements of $A_{2}(\mathbf{C}(z))$ we shall write as $\sum_{i=1}^{n} n_{i}\left[f_{i}(z)\right]$ where $n_{i} \in \mathbf{Z}$ and $f_{i}(x) \in$ 
$\mathbf{C}(z)^{*} \backslash\{1\}$. Let $b_{2}: A_{2}(\mathbf{C}(z)) \rightarrow \mathbf{C}(z)^{*} \wedge \mathbf{C}(z)^{*}$ be given by $b_{2}([f(z)])=f(z) \wedge$ $(1-f(z))$. Let pr: $\mathbf{C}(z)^{*} \wedge \mathbf{C}(z)^{*} \rightarrow \mathbf{C}(z)^{*} \wedge \mathbf{C}(z)^{*} / \mathbf{C}^{*} \wedge \mathbf{C}(z)^{*}$ be the natural projection and let $\beta_{2}:=\operatorname{pr} \circ b_{2}$. We set $\mathscr{B}_{2}(\mathbf{C}(z)):=\operatorname{ker} \beta_{2}$.

We recall one result from [5].

Proposition 4.1. Let $f_{1}, \ldots, f_{N}: X=P^{1}(\mathbf{C}) \backslash\left\{a_{1}, \ldots, a_{n}, \infty\right\} \rightarrow Y=P^{1}(\mathbf{C}) \backslash$ $\{0,1, \infty\}$ be regular functions. Let $n_{1}, \ldots, n_{N}$ be integers. There is a functional equation

$$
\sum_{i=1}^{N} n_{i} \cdot \mathrm{Li}_{2}\left(f_{i}(z)\right)+\overline{\text { l.d.t }}(2)=0
$$

if and only if $\sum_{i=1}^{N} n_{i}\left(f_{i}\right)_{*}=0$ in

$$
\operatorname{Hom}\left(\Gamma^{2} \pi_{1}(X, x) / \Gamma^{3} \pi_{1}(X, x) ; \Gamma^{2} \pi_{1}(Y, y) / \Gamma^{3} \pi_{1}(Y, y)\right),
$$

where $\overline{1 . d . t .}(2)$ is a polynomial in logarithms of rational functions.

The proposition is a special case of Theorem E in [5] or Corollary 2.3 in this paper.

It is a tautological observation that the condition $\sum_{i=1}^{N} n_{i}\left(f_{i}\right)_{*}=0$ is equivalent to the condition $\sum_{i=1}^{N} n_{i}\left[f_{i}(z)\right] \in \operatorname{ker} \beta_{2}$ (see also [5] Theorem 10.8.2). Hence we have the following result.

Proposition 4.2. Let $f_{1}(z), \ldots, f_{N}(z) \in \mathbf{C}(z) \backslash\{0,1\}$ and let $n_{1}, \ldots, n_{N}$ be integers. There is a functional equation

$$
\sum_{i=1}^{N} n_{i} \cdot \operatorname{Li}_{2}\left(f_{i}(z)\right)+\overline{1 . d . t}(2)=0
$$

if and only if $\sum_{i=1}^{N} n_{i}\left[f_{i}(z)\right] \in \operatorname{ker} \beta_{2}$.

Let $\mathscr{B}_{2}^{\text {Abel }}(\mathbf{C}(z)) \subset A_{2}(\mathbf{C}(z))$ be a subgroup of $A_{2}(\mathbf{C}(z))$ generated by the following elements

(3) $\left\{[X \cdot Y]-[X]-[Y]-\left[X \cdot \frac{1-Y}{X-1}\right]-\left[Y \cdot \frac{1-X}{Y-1}\right]\right.$ |

$$
X, Y \in \mathbf{C}(z) \backslash\{0,1\}, X \cdot Y \neq 1\}
$$

(4) $\{[X]+[1 / X] \mid X \in \mathbf{C}(z) \backslash\{0,1\}\}$,

(5) $\{[X]+[1-X] \mid X \in \mathbf{C}(z) \backslash\{0,1\}\}$. 
The terms (3) correspond to the functional equation of Hill and the terms (4) correspond to the equation $\mathrm{Li}_{2}(z)+\mathrm{Li}_{2}(1 / z)+\overline{\text { t.l.d. }}(2)=0$, which can be deduced from the Hill functional equation. The terms (5) correspond to the equation $\mathrm{Li}_{2}(z)+\mathrm{Li}_{2}(1-z)+\overline{\text { t.l.d. }}(2)=0$.

Definition. For any rational function $g(z)=p(z) / q(z)$, where $p(z)$ and $q(z)$ are polynomials without common factors we set $d(g(z)):=\max (\operatorname{deg} p(z)$, $\operatorname{deg} q(z))$.

We say that a rational function $g(z)$ is linear if $g(z)=a \cdot z+b$.

Lemma 4.3. Assume that we have a functional equation

$$
\sum_{i \in I} n_{i} \cdot \mathrm{Li}_{2}\left(h_{i}(z)\right)+\overline{\text { l.d.t }}(2)=0,
$$

where the functions $h_{i}(z)$ are linear and $h_{i}(z) \neq h_{j}(z)$ if $i \neq j$. Then for each $i \in I$ there is $i^{\prime} \in I$ such that $i \neq i^{\prime}, h_{i^{\prime}}(z)=1-h_{i}(z)$ and $n_{i}=n_{i^{\prime}}$.

Proof. The lemma follows immediately from Proposition 4.2. One observes that $\beta_{2}([f])+\beta_{2}([1-f])=0$ for any $f$ in $\mathbf{C}(z)^{*} \backslash\{1\}$. On the other hand, if all $f_{i}(i \in J)$ are linear, $f_{i} \neq f_{i^{\prime}}$ if $i \neq i^{\prime}$ and the intersection $\left\{f_{i}\right\}_{i \in J} \cap\left\{1-f_{i}\right\}_{i \in J}=$ $\emptyset$, then the elements $\left\{\beta_{2}\left[f_{i}\right]\right\}_{i \in J}$ are linearly independent in $\mathbf{C}(z)^{*} \wedge \mathbf{C}(z)^{*} / \mathbf{C}^{*}$ $\wedge \mathbf{C}(z)^{*}$.

THEOREM 4.4. We have $\mathscr{B}_{2}^{\text {Abel }}(\mathbf{C}(z))=\mathscr{B}_{2}(\mathbf{C}(z))$.

Remark. This means that every functional equation of the dilogarithm on $P^{1}(\mathbf{C})$ can be deduced from the Hill functional equation and the equation $\operatorname{Li}_{2}(z)+$ $\mathrm{Li}_{2}(1-z)+\overline{\text { l.d.t. }}(2)=0$.

Proof. For any $f(z)=\alpha \Pi_{i=1}^{n}\left(z-a_{\imath}\right) / \Pi_{i=1}^{m}\left(z-b_{i}\right)$ we have a functional equation

(6) $\operatorname{Li}_{2}(f(z))=\sum_{i, k} \operatorname{Li}_{2}\left(\frac{z-a_{i}}{c_{k}-a_{i}}\right)-\sum_{j, k} \operatorname{Li}_{2}\left(\frac{z-b_{j}}{c_{k}-b_{j}}\right)-\sum_{i, j} \operatorname{Li}_{2}\left(\frac{z-a_{i}}{b_{j}-a_{i}}\right)+\overline{\text { 1.d.t. }}(2)$,

where $c_{k}$ 's are defined by the expression

$$
f(z)-1=\alpha^{\prime} \prod_{k=1}^{r}\left(z-c_{k}\right) / \prod_{j=1}^{m}\left(z-b_{j}\right)
$$


$\alpha, \alpha^{\prime} \in \mathbf{C}^{*}$ and $\Pi_{i=1}^{n}\left(z-a_{i}\right), \Pi_{j=1}^{m}\left(z-b_{j}\right)$ have no common factors (see Theorem $\mathrm{A}$ in [5]).

Let $\mathscr{B}_{2}^{\text {lin }}(\mathbf{C}(z))$ be a subgroup of $A_{2}(\mathbf{C}(z))$ generated by elements

$$
[f(z)]-\sum_{i, k}\left[\frac{z-a_{i}}{c_{k}-a_{i}}\right]+\sum_{j, k}\left[\frac{z-b_{j}}{c_{k}-b_{j}}\right]+\sum_{i, j}\left[\frac{z-a_{i}}{b_{j}-a_{i}}\right]
$$

corresponding to the equation (6) and by elements $[h(z)]+[1-h(z)]$, where $h(z)$ is linear. It is clear that $\mathscr{B}_{2}^{\text {lin }} \subset \mathscr{B}_{2}$ and $\mathscr{B}_{2}^{\text {Abel }} \subset \mathscr{B}_{2}$ (we omit the field $\mathbf{C}(z)$ in the notation).

Let $\sum_{i} n_{i}\left[g_{i}(z)\right] \in \mathscr{B}_{2}$. It follows from (6) that for any $g_{i}(z)$ we have $\left[g_{i}(z)\right]-\sum_{k} \pm\left[h_{i k}(z)\right] \in \mathscr{B}_{2}^{\text {lin }}$ where all $h_{i k}(z)$ are linear. Hence $\sum_{i} n_{i}\left[g_{i}(z)\right]$ $-\sum_{i} n_{i}\left(\sum_{k} \pm\left[h_{i k}(z)\right]\right) \in \mathscr{B}_{2}^{\text {lin }}$ and consequently we have a functional equation $\sum_{i} n_{i}\left(\sum_{k} \pm \mathrm{Li}_{2}\left(h_{i k}(z)\right)\right)+\overline{\text { t.l.d. }}(2)=0$ which we can write in the form

$$
\sum_{\alpha} m_{\alpha} \mathrm{Li}_{2}\left(h_{\alpha}(z)\right)+\overline{\text { t.l.d. }}(2)=0
$$

where all $h_{\alpha}$ are linear and $\alpha \neq \alpha^{\prime}$ implies $h_{\alpha} \neq h_{\alpha^{\prime}}$. It follows from Lemma 4.3 that the equation (7) can be written in the form $\sum_{\beta} n_{\beta} \cdot\left(\mathrm{Li}_{2}\left(h_{\beta}(z)\right)+\mathrm{Li}_{2}(1-\right.$ $\left.\left.h_{\beta}(z)\right)\right)+\overline{\text { l.t.d. }}(2)=0$. The elements $\left[h_{\beta}\right]+\left[1-h_{\beta}\right] \in \mathscr{B}_{2}^{\text {lin }}$ by the definition, hence $\sum_{\alpha} m_{\alpha}\left[h_{\alpha}\right] \in \mathscr{B}_{2}^{\text {lin }}$. This implies that $\sum_{i} n_{i}\left[g_{i}(z)\right] \in \mathscr{B}_{2}^{\text {lin }}$ and therefore we have $\mathscr{B}_{2}^{\text {lin }}=\mathscr{B}_{2}$.

Hence to finish the proof it is sufficient to show that any element of the form

$$
[f(z)]-\sum_{i, k}\left[\left(z-a_{i}\right) /\left(c_{k}-a_{i}\right)\right]+\sum_{j, k}\left[\left(z-b_{j}\right) /\left(c_{k}-b_{j}\right)\right]+\sum_{i, j}\left[\left(z-a_{i}\right) /\left(b_{j}-a_{i}\right)\right]
$$

(where

$$
\begin{aligned}
& f(z)=\left(\alpha \prod_{i=1}^{n}\left(z-a_{i}\right)\right) /\left(\prod_{j=1}^{m}\left(z-b_{j}\right)\right), \\
& f(z)-1=\left(\alpha^{\prime} \prod_{k=1}^{r}\left(z-c_{k}\right)\right) /\left(\prod_{j=1}^{m}\left(z-b_{j}\right)\right),
\end{aligned}
$$

$\alpha, \alpha^{\prime} \in \mathbf{C}^{*}$ and the numerator and the denominator of $f(z)$ have no common factor) belongs to $\mathscr{B}_{2}^{\mathrm{Abel}}$.

By (4) we can assume that $n \geq m$. Let us set $X \cdot Y=f(z)$ and $X=$ $\frac{\left(z-a_{1}\right)}{\left(z-b_{1}\right)} \cdot \frac{\left(c_{1}-b_{1}\right)}{\left(c_{1}-a_{1}\right)}$. Then $X-1=\frac{\left(z-c_{1}\right)\left(a_{1}-b_{1}\right)}{\left(z-b_{1}\right)\left(c_{1}-a_{1}\right)}, Y=\alpha \prod_{i=2}^{n}\left(z-a_{i}\right)$. $\left(c_{1}-a_{1}\right) / \prod_{j=2}^{m}\left(z-b_{j}\right)\left(c_{1}-b_{1}\right)$ and 


$$
Y-1=\frac{\alpha \prod_{i=2}^{n}\left(z-a_{i}\right)\left(c_{1}-a_{1}\right)-\prod_{j=2}^{m}\left(z-b_{j}\right)\left(c_{1}-a_{1}\right)}{\prod_{j=2}^{m}\left(z-b_{j}\right)\left(c_{1}-b_{1}\right)}=\frac{\left(z-c_{1}\right) \mathscr{G}(z)}{\prod_{j=2}^{m}\left(z-b_{j}\right)\left(c_{1}-b_{1}\right)}
$$

where $\operatorname{deg} \mathscr{G}(z) \leq n-2$.

Observe that $d(X), d(Y), d\left(X \cdot \frac{1-Y}{X-1}\right)$ and $d\left(Y \cdot \frac{1-X}{Y-1}\right)$ are strictly smaller than $n$ if $n \geq 2$. We have

$$
\begin{aligned}
X \cdot \frac{1-Y}{X-1} & =-\frac{z-a_{1}}{z-b_{1}} \cdot \frac{c_{1}-b_{1}}{c_{1}-a_{1}} \cdot \frac{\left(z-c_{1}\right) \mathscr{G}(z)}{\prod_{j=2}^{m}\left(z-b_{j}\right)\left(c_{1}-b_{1}\right)} \cdot \frac{\left(z-b_{1}\right)\left(c_{1}-a_{1}\right)}{\left(z-c_{1}\right)\left(a_{1}-b_{1}\right)} \\
& =-\frac{\left(z-a_{1}\right) \mathscr{G}(z)}{\prod_{j=2}^{m}\left(z-b_{j}\right)\left(a_{1}-b_{1}\right)} .
\end{aligned}
$$

Hence $d\left(X \cdot \frac{1-Y}{X-1}\right) \leq n-1$. In the same way one checks for $Y \cdot \frac{1-X}{Y-1}$. If $f(z)$ is a polynomial $\alpha \prod_{i=1}^{n}\left(z-a_{j}\right)$ we set $X=\frac{z-a_{1}}{c_{1}-a_{1}}$. Hence after finite number of steps we get $[f(z)]=\sum_{i} p_{i}\left[g_{i}(z)\right]$ where $d\left(g_{i}(z)\right)=1$. It rests to express $\left[\alpha \cdot \frac{z-a}{z-b}\right]$ by polynomials of degree 1 . We set $X=\frac{z-a}{b-a}, Y=1-\alpha$. Then $X \cdot Y=\frac{(1-\alpha)}{(b-a)} \cdot(z-a), Y \cdot \frac{1-X}{Y-1}=\frac{(\alpha-1)}{\alpha(a-b)} \cdot(z-b)$ and $X \cdot \frac{1-Y}{X-1}$ $=\alpha \cdot \frac{(z-a)}{(z-b)}$.

\section{Exotic analogues of Bloch groups}

In this section we give necessary and sufficient conditions to have a functional equation of iterated integrals in terms of exotic analogues of the Bloch group. We shall work only on a pointed projective line.

Let $\operatorname{Lie}\left(\mathbf{C}(z)^{*}\right)$ be a free Lie algebra on the abelian group $\mathbf{C}(z)^{*}$ (see Section 1). We shall identify an element $f \in \mathbf{C}(z)^{*}$ with $\mathscr{C}_{\mathbf{C}(z)}(f) \in \operatorname{Lie}\left(\mathbf{C}(z){ }^{*}\right)$. Let $I \subset$ $\operatorname{Lie}\left(\mathbf{C}(z)^{*}\right)$ be a Lie ideal consisting of all brackets $\left[\ldots\left[f_{1}, f_{2}\right] \ldots\left[\ldots, f_{k}\right]\right.$ ...] such that at least one $f_{i}$ is in $\mathbf{C}^{*}$. Let $L\left(\mathbf{C}(z)^{*}\right):=\operatorname{Lie}\left(\mathbf{C}(z)^{*}\right) / I$. Observe that $L\left(\mathbf{C}(z)^{*}\right)$ is a free Lie algebra on the set $\{(z-a) \mid a \in \mathbf{C}\}$.

Let $\mathbf{X}=\mathbf{P}^{1}(\mathbf{C}) \backslash\left\{a_{1}, \ldots, a_{n}, \infty\right\}$ and let $\mathbf{Y}=\mathbf{P}^{1}(\mathbf{C}) \backslash\left\{b_{1}, \ldots, b_{m}, \infty\right\}$. We 
set

$$
\mathscr{B}(\mathbf{Y}):=\bigoplus_{f \in \mathbf{C}(z) \backslash\left\{b_{1}, \ldots, b_{m}\right\}} \mathbf{Z},
$$

the free abelian group on the set $\mathbf{C}(z) \backslash\left\{b_{1}, \ldots, b_{m}\right\}$. The generator corresponding to $f$ we shall denote by $[f]$. For any homogeneous element $e \in \operatorname{Lie}(H(\mathbf{Y}))$,

$$
e=\sum_{i} \alpha_{i}\left[\ldots\left[B_{i_{1}} \ldots\left[\ldots, B_{i_{k}}\right] \ldots\right]\right. \text {, }
$$

where $B_{j}=\left(\frac{d z}{z-b_{j}}\right)^{*}$ we define a map

$$
b_{Y}(e): \mathscr{B}(\mathbf{Y}) \rightarrow L\left(\mathbf{C}(z)^{*}\right)
$$

by the formula

$$
b_{Y}(e)([f])=\sum_{i} \alpha_{i}\left[\ldots\left[f-b_{i_{1}} \ldots\left[\ldots, f-b_{i_{k}}\right] \ldots\right] .\right.
$$

Let us fix an ordering $B_{1}=\left(\frac{d z}{z-b_{1}}\right)^{*}, B_{2}, \ldots, B_{m}$ of the base of $H(\mathbf{Y})$. Then there is a canonical base $\mathscr{B}=\left\{e_{i}\right\}_{i \in I}$ of $\operatorname{Lie}(H(Y))$ given by basic Lie elements (see [6]). Let $\left\{e_{i}^{*}\right\}_{i \in I}$ be the dual linear forms.

Theorem 5.1. Let $e_{1}, \ldots, e_{n} \in \mathscr{B}$ be basic Lie elements of degree $n$. Let $f_{1}, \ldots$, $f_{n}: \mathbf{X} \rightarrow \mathbf{Y}$ be regular maps. Let $n_{1}, \ldots, n_{N}$ be integers. Let $\gamma$ be a path in $\mathbf{X}$ from $x$ to $z$. The following conditions are equivalent.

$$
\sum_{i=1}^{N} n_{i} \cdot e_{i}^{*}\left(L_{\mathbf{Y}}\left(f_{i}(z) ; f_{i}(x), f_{i}(\gamma)\right)=0 ;\right.
$$

ii)

$$
\sum_{i=1}^{N} n_{i} \cdot e_{i}^{*} \circ\left(f_{i}\right)_{*}=0 \text { in } \operatorname{Hom}\left(\Gamma^{n} \pi(\mathbf{X}) / \Gamma^{n+1} \pi(\mathbf{X}) ; \mathbf{C}\right) \text {; }
$$

iii)

$$
\sum_{i=1}^{N} n_{i} \cdot b_{\mathbf{Y}}\left(e_{i}\right)\left(\left[f_{i}\right]\right)=0
$$

Proof. It follows from Corollary 2.2 that conditions i) and ii) are equivalent. Hence we must show that ii) and iii) are equivalent. Let $e=\left[\ldots\left[B_{i_{1}}, \ldots[\ldots\right.\right.$, $B_{i_{k}}$ ]...] be a basic Lie element of degree $n$ and let $e^{*}$ be its dual. Let $f: \mathbf{X} \rightarrow \mathbf{Y}$ be a regular map. The map $f$ and $e^{*}$ induce

$$
\operatorname{Lie}(H(\mathbf{X})) \stackrel{f_{*}}{\rightarrow} \operatorname{Lie}(H(\mathbf{Y})) \stackrel{e^{*}}{\rightarrow} \mathbf{C} .
$$

Let us set $\mathbf{C}^{\vee}:=\operatorname{Hom}(\mathbf{C}, \mathbf{C})$. Passing to dual objects we get a map

$$
\operatorname{Lie}\left(A^{1}(\mathbf{X})\right) \stackrel{f^{*}}{\leftarrow} \operatorname{Lie}\left(A^{1}(\mathbf{Y})\right) \stackrel{\left(e^{*}\right)^{\vee}}{\longleftarrow} \mathbf{C}^{\vee} .
$$


Observe that the condition ii) is equivalent to the condition

$$
\sum_{i=1}^{N} n_{i} \cdot\left(f_{i}\right)^{*} \circ\left(e_{i}^{*}\right)^{\vee}=0 \text { in } \operatorname{Hom}\left(\mathbf{C}^{\vee}, \operatorname{Lie}\left(A^{1}(\mathbf{X})\right)\right) .
$$

Observe that $f^{*}\left(\frac{d z}{z-b_{i}}\right)=\frac{f^{\prime}(z)}{f(z)-b_{i}} d z$ and $\left(e^{*}\right)^{\vee}\left(\mathrm{id}_{\mathbf{C}}\right)=\left[\ldots \frac{d z}{z-b_{i_{1}}} \ldots[\ldots\right.$, $\left.\left.\frac{d z}{z-b_{i_{k}}}\right] \ldots\right]$. Let us define maps

$$
i_{\mathbf{X}}: \operatorname{Lie}\left(A^{1}(\mathbf{X})\right) \rightarrow L\left(\mathbf{C}(z)^{*}\right) \text { and } i_{\mathbf{Y}}: \operatorname{Lie}\left(A^{1}(\mathbf{Y})\right) \rightarrow L\left(\mathbf{C}(z)^{*}\right)
$$

by the formula $i_{\mathbf{x}}\left(\frac{d z}{z-a_{i}}\right)=\left(z-a_{i}\right)$. Observe that the diagram

$$
\begin{array}{cccc}
\operatorname{Lie}\left(A^{1}(\mathbf{X})\right) & \stackrel{\left(f^{*}\right)}{\leftarrow} & \operatorname{Lie}\left(A^{1}(\mathbf{Y})\right) & \stackrel{\left(e^{*}\right)^{\vee}}{\leftarrow} \mathbf{C}^{\vee} \\
\downarrow i_{\mathbf{X}} & \stackrel{\left(f^{\sharp}\right)}{\leftarrow} & \downarrow i_{\mathbf{Y}} & \swarrow \tilde{e}:=i_{\mathbf{Y}} \circ\left(e^{*}\right)^{\vee} \\
L\left(\mathbf{C}(z)^{*}\right) & \leftarrow & L\left(\mathbf{C}(z)^{*}\right) &
\end{array}
$$

commutes, where $f^{\#}(z-a)=f(z)-a$ and $\tilde{e}\left(\mathrm{id}_{\mathbf{C}}\right)=\left[\ldots\left[z-b_{i_{1}}, \ldots[\ldots, z-\right.\right.$

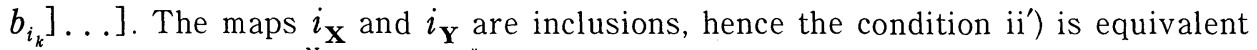
to the condition $\sum_{i=1}^{N} n_{i} \cdot\left(f_{i}\right)^{\#} \circ \tilde{e}_{i}=0$. This last condition is equivalent to the condition $\sum_{i=1}^{N} n_{i} \cdot\left(f_{i}\right)^{\#}\left(\tilde{e}_{i}\left(\mathrm{id}_{\mathbf{C}}\right)\right)=0$. Observe that $\left(f_{i}\right)^{\#}\left(\tilde{e}_{i}\left(\operatorname{id}_{\mathbf{C}}\right)\right)=b_{\mathbf{Y}}\left(e_{i}\right)\left[f_{i}\right]$. Hence we get that ii) and iii) are equivalent.

\section{REFERENCES}

[1] P. Deligne, Théorie de Hodge II, Publ. Math. IHES, No 40 (1971), 5-58.

[2] C. J. Hill, Specimen exercitti analytici, functionum integralum $\int_{0}^{x} \frac{d x}{x} \log (1+$ $\left.2 x \cos \alpha+x^{2}\right)$ tum secundum amplitudinem, tum secundum modulum comparandi exhibentis, Lund, 9 (1830).

[ 3 ] H. Hironaka, Resolution of singularities of an algebraic variety over a field of characteristic zero I II, Ann. of Math., $79(1964) \mathrm{n}^{\circ} 1$ and $\mathrm{n}^{\circ} 2$.

4 ] L. Lewin, The Evolution of the Ladder Concept, 1-10 in Structural Properties of Polylogarithms, Mathematical Surveys and Monographs, vol. 37, AMS, 1991.

[5] Z. Wojtkowiak, The Basic Structure of Polylogarithmic Functional Equations, 205-231 in Structural Properties of Polylogarithms, L. Lewin, Editor, Mathematical Surveys and Monographs, vol. 37, AMS, 1991.

[6] W. Magnus, A. Karrass and D. Solitar, Combinatorial Group Theory, Pure and Applied Mathematics, XIII, Interscience Publ, 1966. 
Universite de Nice-Sophia Antipolis

Departement de Mathématiques

Laboratoire Jean Alexandre Dieudonné

U.R.A. au C.N.R.S., No 168

Parc Valrose-B.P. $N^{\circ} 71$

06108 Nice Cedex 2, France

Research Institute

for Mathematical Sciences

Kyoto University

Kitashirakawa, Sakyo-ku,

Kyoto 606, Japan 